\title{
CALIDAD NUTRICIONAL Y DEGRADABILIDAD RUMINAL DE LA PLANTA DEL GUINEO NEGRO (Musa AAA) ${ }^{1}$
}

\author{
Pablo Chacón-Hernández ${ }^{2}$, Carlos Boschini-Figueroa ${ }^{2}$, Ricardo Russo-Andrade ${ }^{2}$
}

\section{RESUMEN}

Calidad nutricional y degradabilidad ruminal de la planta del guineo negro (Musa AAA). El objetivo del trabajo fue evaluar el valor alimenticio de un banco forrajero de guineo negro (Musa AAA). El estudio se llevó a cabo en la Estación Experimental Alfredo Volio Mata de la Universidad de Costa Rica, localizada en la provincia de Cartago, Costa Rica. El estudio fue realizado durante los meses de mayo a noviembre del año 2013 y se empleó un diseño de bloques completos al azar. Se obtuvieron valores promedio en la planta entera de $5,67 \%$ de materia seca, $6,50 \%$ de proteína cruda, $53,91 \%$ de fibra neutro detergente, $35,67 \%$ en fibra ácido detergente, $7,61 \%$ de lignina, 28,07\% de celulosa, $18,23 \%$ de hemicelulosa, $1,95 \%$ de extracto etéreo, 19,30\% de cenizas, $4,63 \%$ de proteína cruda fijada a la fibra neutro detergente y 25,32\% de carbohidratos no fibrosos; además, para todos los componentes bromatológicos se encontraron diferencias estadísticamente significativas $(\mathrm{p}<0,05)$ de acuerdo con la parte de la planta muestreada. En los análisis de degradabilidad ruminal, se encontraron valores de fracción soluble de 18,38\% a 47,43\%, fracción degradable de $33,45 \%$ a $45,76 \%$, velocidades de degradación de $1,65 \% / \mathrm{h}$ a $7,51 \% / \mathrm{h}$ y porcentajes potencialmente degradables de $64,14 \%$ a $82,86 \%$, según la parte de la planta muestreada. Según los datos analizados, la planta de Guineo Negro podría ser utilizada para alimentación de rumiantes en los lugares donde se encuentre disponible.

\begin{abstract}
Nutritional quality and ruminal degradability of banana (Musa AAA) plant. The objective of the study was to evaluate the nutritional value of a Musa AAA fodder bank. The study was conducted at the Alfredo Volio Mata Experimental Station of the University of Costa Rica. It took place from May to November 2013 using a complete random block design. Mean values for the whole plant were of $5.67 \%$ of dry matter, $6.50 \%$ of crude protein, $53.91 \%$ of neutral detergent fiber, $35.67 \%$ of acid detergent fiber, $7.61 \%$ of lignin, $28.07 \%$ of cellulose, $18.23 \%$ of hemicellulose, $1.95 \%$ of ether extract, $19,30 \%$ of ash, $4.63 \%$ of crude protein attached to the neutral detergent fiber, and $25.32 \%$ of non- fibrous carbohydrates. Besides, statistical differences were found $(\mathrm{p}<0.05)$ for all bromatological components, according to the sampled plant part. The analysis of ruminal degradability showed values ranging from $18.38 \%$ to $47.43 \%$ for the soluble fraction, from $33.45 \%$ to $45.76 \%$ for the degradable fraction, from $1.65 \% / \mathrm{h}$ to $7.51 \% / \mathrm{h}$ for the degradation speed, and from $64.14 \%$ to $82.86 \%$ the potentially degradable percentage, depending on the part of the plant sampled. According to analyzed data, Musa AAA plants could be used for feeding ruminants where available.
\end{abstract}

Keywords: forage, bromatology, fodder bank.

Palabras clave: forraje, bromatología, banco forrajero.

\footnotetext{
Recibido: 23 de julio, 2015. Aceptado: 24 de setiembre, 2015. Este trabajo formó parte del proyecto 737-B1-074, inscrito en la Vicerrectoría de Investigación de la Universidad de Costa Rica, San José, Costa Rica.

2 Universidad de Costa Rica, Facultad de Ciencias Agroalimentarias, Estación Experimental Alfredo Volio Mata, Cartago, Costa Rica. pablo. chacon@ucr.ac.cr (autor para correspondencia), carlos.boschini@ucr.ac.cr, ricardo.russo@ucr.ac.cr
} 


\section{INTRODUCCIÓN}

Anualmente, a nivel mundial se produce una cantidad considerable de materia orgánica en los diversos procesos de la producción agrícola (Costa et al., 2011), pero solo una parte de esa producción es aprovechada directamente (Villalba et al., 2011). El cultivo de las diversas variedades de musáceas existentes es el segundo en magnitud después de los cítricos, contribuyendo a un $16 \%$ del total de fruta producida.

Desde el punto de vista genético, el origen de los ecotipos actuales de musáceas se dio por medio de cruces de Musa acuminata Colla y Musa balbisiana Colla, las cuales se denotan como los genomas A y B, respectivamente, pudiendo verse híbridos diploides, triploides y tetraploides por la hibridación entre ambas especies (Mohapatra et al., 2010). Asimismo, el "pool” germoplásmico del género Musa, se ha diversificado hasta grados muy complejos por mutaciones somáticas que se han dado de forma guiada o natural (Osuji et al., 1997). En el caso del guineo negro, este pertenece genéticamente a un grupo puro triploide AAA proveniente de la Musa acuminata Colla (Mohapatra et al., 2010).

En las plantaciones de musáceas o zonas aledañas, el uso de la biomasa vegetal como forraje para alimentación animal podría constituir una alternativa económica para los agricultores (Mohapatra et al., 2010), ya que lo único cosechado es el fruto para la venta comercial, y un $70-80 \%$ de la producción no es aprovechada (Mazzeo et al., 2010), quedando muchas veces en el campo hasta su descomposición natural o incineración por parte del agricultor (García y Martínez, 1999), lo que genera muchas veces problemas de contaminación (Villalba et al., 2011). Dicha situación obliga a implementar sistemas de manejo que disminuyan el impacto ambiental generado por el cultivo (Mazzeo et al., 2010; Costa et al., 2011).

En Costa Rica muchos productores pecuarios han recurrido de forma empírica al uso de subproductos y residuos de cosecha, como alternativa para la alimentación, p. ej. el pseudo-tallo de las musáceas, esto se realiza con el fin de reducir los costos de producción (Dormond et al., 2011) y disminuir la dependencia de insumos externos (De-la-Cruz y Gutiérrez, 2006).

Desde el punto de vista práctico, la limitante actual en el uso del vástago como fuente alimenticia radica en el hecho de que es el residuo menos estudiado en las plantaciones de musáceas (Dormond et al., 2000; Amaral et al., 2008), además, la composición bromatológica de un forraje no es suficiente para determinar la calidad de un material, ya que al mismo tiempo su aprovechamiento está determinado por el grado de digestibilidad en el animal (Weiss et al., 1992).

El objetivo de este trabajo fue evaluar el valor alimenticio de un banco forrajero de guineo negro (Musa sp.).

\section{MATERIALES Y MÉTODOS}

\section{Ubicación del experimento}

El experimento se llevó a cabo en la Estación Experimental Alfredo Volio Mata, de la Universidad de Costa Rica, la cual se encuentra ubicada en El Alto de Ochomogo, distrito de San Rafael del cantón de La Unión en la provincia de Cartago, Costa Rica, a 1542 msnm. Las condiciones climatológicas de la zona comprenden una precipitación anual media de 1465,9 $\mathrm{mm}$, distribuidos principalmente en la época lluviosa que va de mayo a noviembre; una humedad relativa media del $88 \%$ y una temperatura que va de los 13,5 ${ }^{\circ} \mathrm{C}$ a los $22,2{ }^{\circ} \mathrm{C}$ con un promedio anual de 17,9 ${ }^{\circ} \mathrm{C}$ (Instituto Meteorológico Nacional, comunicación personal, 2013). Las coordenadas geográficas correspondientes a dicho centro experimental son $9^{\circ} 55^{\prime} 10 "$ latitud norte y 8357'20" longitud oeste (Chacón-Hernández, 2008), el suelo es de origen volcánico, clasificado como Typic distrandepts, caracterizado por buen drenaje y fertilidad media; asimismo el ecosistema es considerado como bosque húmedo montano bajo (Vásquez, 1982).

\section{Descripción del material de estudio}

Las muestras analizadas fueron tomadas de un banco forrajero de guineo negro (Musa AAA), establecido en la estación experimental durante la época lluviosa del año 2013, y en el cual las cepas de las plantas fueron sembradas con una disposición de marco real de dos metros de lado. Además, a dicho banco no se le realizó ningún tipo de poda, cosecha o aplicación de fertilizantes desde un año antes del inicio del periodo experimental. 


\section{Definición de parcelas y diseño experimental}

Con el fin de eliminar las posibles variaciones generadas por la ubicación de las plantas dentro del banco forrajero, debidas a diferente radiación, suelo, humedad, entre otras, y determinar la calidad nutricional de las diferentes secciones del pseudotallo y la hoja, se utilizó un diseño experimental de bloques completos al azar (Steel y Torrie, 1988), con tres bloques y cinco tratamientos. Cada bloque fue definido de forma aleatoria dentro del banco forrajero, con dimensiones de seis metros de ancho por ocho metros de largo, de forma que dentro de cada uno se tuviera un máximo de doce cepas para realizar los muestreos $\left(P_{i}\right)$. Las partes de la planta muestreadas, correspondientes a tres secciones del pseudo-tallo y dos partes de la hoja, se definieron como los cinco tratamientos analizados $\left(T_{j}\right)$. El modelo estadístico utilizado para el análisis de la información correspondió a:

$$
y_{i j k}=\mu+P_{i}+T_{j}+e_{i j k}
$$

\section{Proceso de muestreo}

Se realizó un muestreo en el cual se tomó de forma aleatoria cinco de los vástagos más aptos para la cosecha en cada parcela, los cuales todavía no habían alcanzado su época reproductiva, para un total de quince pseudo-tallos; tomando únicamente como criterio de selección para el muestreo las dimensiones de las plantas, seleccionando siempre las de mayor tamaño. El corte fue realizado a una altura de 20 a 25 $\mathrm{cm}$ sobre el nivel del suelo; inmediatamente después se dividió cada planta en dos secciones correspondientes a hojas y vástago o palote, y se les realizó el corte en el punto de bifurcación de las hojas.

Con el fin de determinar la calidad nutricional de las diferentes partes de la planta del guineo negro, cada vástago fue dividido en tres secciones correspondientes a la parte basal, media y apical del mismo, de las cuales se tomó una muestra que fue enviada al laboratorio para su análisis; además, de cada pseudo-tallo se recolectó una muestra de hojas las cuales fueron separadas en dos partes: lámina y peciolo, para ser enviadas al laboratorio de bromatología de la estación experimental.

\section{Análisis de muestras}

Los análisis de laboratorio de las muestras fueron desarrollados en el laboratorio de bromatología de la Estación Experimental Alfredo Volio Mata. A todas las muestras recolectadas, se les realizó análisis químicos de: materia seca (MS; AOAC 934.01); proteína cruda (PC) por el método de Kjeldahl (AOAC 954.01); extracto etéreo (EE; AOAC 920.39) y cenizas totales (CEN; AOAC 935.12) según los procedimientos establecidos por la AOAC (2000); además, fibra neutro detergente (FND), fibra ácido detergente (FAD) y la lignina (LIG) por el método de Goering y VanSoest (1970) y Van-Soest et al. (1991); proteína cruda incrustada en la fibra neutro detergente (PCIFND) por medio de un análisis de FND según los protocolos de Goering y Van-Soest (1970) y Van-Soest et al. (1991), seguido de un análisis de PC sobre el residuo obtenido según el método de Kjeldahl (AOAC, 2000); hemicelulosa (HEMI) y celulosa (CEL), los cuales se estimaron por diferencia de las fracciones correspondientes; finalmente, se determinó la cantidad de carbohidratos no fibrosos (CNF) mediante la siguiente fórmula (NRC, 2001):

$$
\mathrm{CNF}=100-((\mathrm{FND}-\mathrm{PCIFND})+\mathrm{PC}+\mathrm{EE}+\mathrm{CEN})
$$

donde:

$\mathrm{CNF}=$ carbohidratos no fibrosos, $\%$

FND = fibra neutro detergente, $\%$

PCIFND = proteína cruda incrustada en la fibra neutro detergente, $\%$

$\mathrm{PC}=$ proteína cruda, $\%$

$\mathrm{EE}=$ extracto etéreo, \%

$\mathrm{CEN}=$ cenizas, $\%$

Se realizaron pruebas de degradabilidad ruminal según la metodología descrita por Ørskov et al. (1980), para lo cual se utilizaron dos animales híbridos JerseyReyna fistulados ruminalmente, de los cuales ninguno se encontraba en producción y estaban alimentados únicamente con pasto Estrella Africana (Cynodon nlemfluensis) desde una semana antes y durante de la incubación.

\section{Análisis de resultados}

Los valores determinados en laboratorio para las muestras de vástago fueron sometidos al análisis de 
varianza con el PROC ANOVA del paquete estadístico SAS (1985). Aquellas variables que mostraron diferencias importantes entre medias, fueron sometidas a la prueba de Duncan para su segregación estadística.

Los resultados de degradabilidad ruminal fueron ajustados con el mismo paquete estadístico (SAS, 1985), mediante el algoritmo de Marquardt (Vargas, 2005; Chacón-Hernández y Vargas-Rodríguez, 2009):

$$
y_{i}=a+b\left(1-e^{-c^{*} t_{i}}\right)
$$

donde:

$\mathrm{y}_{\mathrm{i}}=$ degradación del material en el iésimo tiempo de incubación, \%

$$
\begin{aligned}
& \mathrm{a}=\text { fracción soluble }, \% \\
& \mathrm{~b}=\text { fracción degradable, } \% \\
& \mathrm{e}=\text { base de los logaritmos neperianos } \\
& \mathrm{c}=\text { tasa de degradación, } \% \text { hora } \\
& \mathrm{t}_{\mathrm{i}}=\text { tiempo de incubación ruminal, horas }
\end{aligned}
$$

\section{RESULTADOS Y DISCUSIÓN}

\section{Composición nutricional}

No se encontró diferencias significativas entre parcelas $(p>0,05)$ para ninguno de los componentes evaluados en el análisis bromatológico del banco de guineo negro, aunque si fueron determinadas diferencias significativas $(\mathrm{p}<0,05)$ de acuerdo con la sección de la planta evaluada (Cuadro 1).

\section{Materia seca (MS)}

El contenido de materia seca (MS) aumentó significativamente $(\mathrm{p}<0,05)$ conforme se subió a través de las diferentes secciones de la planta (Cuadro 1), lo cual pudo deberse a la mayor proporción de tejido de transporte presente en tejidos fisiológicamente de mayor edad y proporción de aerénquima, o a una exportación de compuestos solubles desde las partes

\begin{tabular}{|c|c|c|c|c|c|c|c|c|c|c|c|c|c|c|c|c|}
\hline \multirow{3}{*}{$\begin{array}{l}\text { Componente } \\
\text { bromatológico }\end{array}$} & \multicolumn{14}{|c|}{ Parte de la planta } & \multicolumn{2}{|c|}{ Planta entera } \\
\hline & \multicolumn{8}{|c|}{ Pseudo-tallo } & \multicolumn{6}{|c|}{ Hoja } & \multirow[b]{2}{*}{ Promedio } & \multirow[b]{2}{*}{ DE } \\
\hline & Basal & DE & Medial & & DE & Apical & & DE & Vaina & & DE & Lámina & & DE & & \\
\hline MS, \% & $3,93 \mathrm{e}$ & 0,16 & 4,97 & d & 0,43 & 6,13 & $\mathrm{c}$ & 0,62 & 10,38 & $\mathrm{~b}$ & 0,36 & 20,68 & $\mathrm{a}$ & 0,54 & 5,67 & 0,45 \\
\hline PC, \% & $5,61 \mathrm{~b}$ & 1,07 & 5,87 & $\mathrm{~b}$ & 0,98 & 5,99 & $\mathrm{~b}$ & 0,43 & 5,56 & $\mathrm{~b}$ & 0,83 & 19,82 & $\mathrm{a}$ & 0,65 & 6,50 & 1,01 \\
\hline FND, $\%$ & $50,63 \mathrm{~d}$ & 4,72 & 55,71 & $\mathrm{c}$ & 5,49 & 54,29 & $\mathrm{c}$ & 5,13 & 61,34 & $\mathrm{~b}$ & 1,25 & 68,11 & $\mathrm{a}$ & 1,34 & 53,91 & 4,94 \\
\hline FAD, $\%$ & $33,82 \mathrm{c}$ & 4,28 & 37,50 & $\mathrm{~b}$ & 4,68 & 36,15 & bc & 4,17 & 44,24 & a & 1,21 & 36,24 & $\mathrm{bc}$ & 0,78 & 35,68 & 4,41 \\
\hline LIG, \% & $6,84 \mathrm{c}$ & 1,81 & 7,57 & $\mathrm{c}$ & 2,32 & 7,15 & $\mathrm{c}$ & 0,92 & 9,90 & $\mathrm{~b}$ & 1,26 & 14,79 & $\mathrm{a}$ & 1,26 & 7,61 & 1,59 \\
\hline CEL, $\%$ & $26,98 \mathrm{c}$ & 2,63 & 29,94 & b & 3,29 & 29,01 & $\mathrm{bc}$ & 3,42 & 34,34 & a & 0,68 & 21,46 & d & 1,50 & 28,07 & 2,90 \\
\hline HEMI, \% & $16,81 \mathrm{c}$ & 1,76 & 18,21 & $\mathrm{~b}$ & 1,85 & 18,14 & $\mathrm{~b}$ & 1,79 & 17,10 & $\mathrm{bc}$ & 0,60 & 31,86 & $\mathrm{a}$ & 0,76 & 18,23 & 1,51 \\
\hline $\mathrm{EE}, \%$ & $1,62 \mathrm{~b}$ & 0,14 & 1,96 & b & 0,82 & 2,07 & $\mathrm{~b}$ & 0,62 & 1,85 & $\mathrm{~b}$ & 1,85 & 4,27 & $\mathrm{a}$ & 0,49 & 1,95 & 0,16 \\
\hline CEN, \% & $19,30 \mathrm{a}$ & 2,70 & 16,73 & $\mathrm{~b}$ & 2,28 & 13,99 & $\mathrm{c}$ & 2,12 & 11,74 & d & 0,84 & 9,12 & $\mathrm{e}$ & 0,28 & 16,95 & 2,55 \\
\hline PCIFND, $\%$ & $3,83 \mathrm{~b}$ & 0,46 & 3,72 & b & 0,36 & 3,83 & $\mathrm{~b}$ & 0,40 & 3,89 & $\mathrm{~b}$ & 0,69 & 19,26 & $\mathrm{a}$ & 0,82 & 4,63 & 0,34 \\
\hline $\mathrm{CNF}, \%$ & 26,68 a & 6,55 & 23,45 & $\mathrm{a}$ & 7,55 & 27,49 & $\mathrm{a}$ & 7,24 & 23,41 & $\mathrm{a}$ & 1,72 & 17,96 & $\mathrm{~b}$ & 0,73 & 25,32 & 6,94 \\
\hline
\end{tabular}
fisiológicamente más viejas a las jóvenes (Maurice et

Cuadro 1. Composición bromatológica de las diferentes partes de la planta de una muestra de quince plantas de musáceas (Musa sp.). Cartago, Costa Rica. 2013.

Table 1. Bromatological composition from the different parts of the plant of fifteen samples of musacea plants (Musa sp.). Cartago, Costa Rica. 2013.

DE: desviación estándar; MS: materia seca; PC: proteína cruda; FND: fibra neutro detergente; FAD: fibra ácido detergente; LIG: lignina; CEL: celulosa; HEMI: hemicelulosa; EE: extracto etéreo; CEN: cenizas totales; PCIFND: proteína cruda incrustada en la fibra neutro detergente; CNF: carbohidratos no fibrosos / DE: Standard deviation; MS: dry matter; PC: crude protein; FND: neutral detergent fiber; FAD: acid detergent fiber; LIG: lignin; CEL: cellulose; HEMI: hemicellulose; CEN: total ash; PCIFND: crude protein in neutral detergent insoluble; CNF: non fibrous carbohydrates.

Letras diferentes en las columnas indican diferencias significativas $(\mathrm{p}<0,05)$ entre las secciones de la planta para un mismo componente bromatológico / Different letters in columns indicate statistical differences $(\mathrm{p}<0.05)$ among the parts of the plant for each nutritional component. 
al., 1997; Avila et al., 2010); inclusive el cambio en el contenido de MS al pasar de la sección basal a la media y de esta última a la apical, constituyó cambios de $3,93 \%$ y $4,97 \%$ de la MS, respectivamente.

Los porcentajes de MS encontrados fueron similares a los reportados por Oliveira et al. (2007), quienes encontraron en Musa acuminata Colla, var. Cavendish un $11,0 \%, 25,7 \%$ y $8,3 \%$ de materia seca en peciolos, láminas y pseudo-tallos, respectivamente. Los valores obtenidos también fueron similares al $10,1 \%$ de MS que indican Marie-Magdeleine et al. (2010) al analizar muestras de la planta entera de Musa x paradisiaca. Además, la literatura indica que únicamente las hojas de musáceas alcanzan valores superiores al 20\% de MS, mientras que los pseudotallos o palotes, normalmente se encuentran bajo el 10\% (Katongole et al., 2008), lo cual es coincidente con lo determinado en las muestras analizadas (Cuadro 1).

Al comparar los contenidos de MS del material analizado de la planta entera (Cuadro 1), con el de forrajes de corta comúnmente utilizados, como Pennisetum purpureum o el sorgo negro forrajero, para los cuales se han reportado valores mínimos de $12 \%$ cuando son cosechados a una edad de 70 y 91 días, respectivamente (Araya y Boschini, 2005; Boschini y Elizondo, 2005), el contenido de MS en las musáceas es considerablemente más bajo, aunque este material podría también tomarse como una opción para el suministro de agua al animal durante la época seca en ciertas zonas climatológicas, donde se tienen problemas de escasez de agua.

\section{Proteína cruda (PC)}

En un estudio en el que se analizaron los contenidos de nitrógeno en cinco variedades de musáceas, se encontró una concentración máxima de proteína en las hojas de $16,06 \%$, mientras que la más baja fue 2,31\% en el vástago (Hernández et al., 2009); los valores determinados en ese estudio fueron más bajos que los presentados en el Cuadro 1. Sin embargo, si los valores obtenidos en este ensayo se comparan con el $21,6 \%$ en hojas y $9,7 \%$ en vainas de la hoja en el pseudo-tallo de Musa acuminata (Katongole et al., 2008), fueron ligeramente inferiores. Estas variaciones pudieron deberse a la estrecha relación entre el clon, tipo de suelo, sistema de cultivo y manejo de las plantaciones de musáceas con el contenido de nitrógeno (García y Martínez, 1999).
La concentración de PC determinada en las diferentes secciones del vástago, presentó valores que fueron más bajos a los encontrados en otros forrajes de corta como el sorgo, para el cual se ha reportado un valor promedio de 12,66\% luego de 77 días de crecimiento (Vargas, 2004); o el pasto Pennisetum purpureum cv. King Grass cosechado a 60 días de rebrote con 9,56\% (Chacón y Vargas, 2010). Además, las cantidades determinadas en este estudio fueron inferiores al 7\% de PC mínimo que deben poseer los materiales utilizados en alimentación de rumiantes para mantener un adecuado ambiente ruminal (Sánchez et al., 1998).

La mayor cantidad de compuestos nitrogenados, carbohidratos y minerales suelen encontrarse en las zonas fisiológicamente más activas en términos de crecimiento (Maurice et al., 1997), lo cual puede explicar la diferencia significativa observada en el contenido de $\mathrm{PC}$ en las diferentes secciones de la planta.

La planta de la morera, a una edad de setenta días de crecimiento, puede tener un 7,75\% de PCIFND de un total de PC de $20,88 \%$, lo cual corresponde a un $37,11 \%$ ligado a la fibra (Boschini-Figueroa, 2006), valor que es considerablemente más bajo a lo determinado en este estudio (Cuadro 1), donde más del $60 \%$ de los compuestos nitrogenados se encuentran ligados a la fibra, e inclusive llegan a ser un $97,17 \%$ de la PC en el caso de la lámina de la hoja, esto indicó que la mayoría del nitrógeno en el material analizado se encontraba en la forma de proteínas ligadas a la pared celular, de la cual una parte puede ser aprovechada según se libere y se haga disponible por la digestión ruminal, mientras que existe una fracción ligada a la FAD que no será aprovechada por el animal (NRC, 2001).

\section{Fibra}

El valor de fibra neutro detergente (FND) en las hojas de Musa x paradisiaca fue de 68,0\% (Marie-Magdeleine et al., 2010), valor que no difiere de forma considerable con lo determinado en las muestras analizadas para esta fracción (Cuadro 1), otros experimentos reportan un valor de $67,2 \%$ y 69,5\% de FND en pseudo-tallos de Musa cavendishi (Poyyamozhi y Kadrivel, 1986; Viswanathan et al., 1989), valores que fueron cercanos a lo encontrado para la lámina de la hoja en las muestras analizadas.

Un 47,22\% de FAD fue reportado por Aziz et al. (2011); 45,0\% para la misma fracción fue indicada 
por Marie-Magdeleine et al. (2010), siendo en general estos valores más elevados a los obtenidos en las muestras de vástago y las láminas de las hojas en este estudio. Katongole et al. (2008) determinaron valores de lignina en pesudo-tallos y hojas de $10,2 \%$ y $12,4 \%$, respectivamente, siendo mayor lo encontrado en el vástago e inferior a la lámina (Cuadro 1); asimismo, estos porcentajes elevados podrían generar un problema de consumo, ya que la lignina es indigestible y en niveles elevados disminuye la ingesta voluntaria (NRC, 2001; Aziz et al., 2011).

Al analizar los contenidos de celulosa de las diferentes partes morfológicas de las musáceas, se obtuvieron proporciones de $34,0-40,0 \%, 31,0 \%$ y 20,4\% en el pseudo-tallo, peciolo y lámina de la hoja, respectivamente (Mohapatra et al., 2010); estos valores fueron similares a los observados en este estudio en las hojas, pero menores para las tres secciones analizadas del palote, las cuales a su vez presentaron valores superiores al 22,5\% reportado para Musa acuminata (Katongole et al., 2008).

Se ha encontrado un $18,56 \%$ de hemicelulosa (HEMI) en el pseudo-tallo de musáceas (Aziz et al., 2011), valor que fue cercano a lo que se reportó en el presente ensayo (Cuadro 1). Sin embargo, los resultados de holocelulosa de 60-65\%, 62,7\% y $32,1 \%$ para pseudo-tallo, peciolo y lámina de la hoja, respectivamente, indicados por Mohapatra et al. (2010), sugieren proporciones de HEMI que difieren de manera considerable a lo determinado en este experimento, al ser la holocelulosa la suma de la CEL y la HEMI (Rabemanolontsoa y Saka, 2012).

Las variaciones en la composición de las fracciones de la fibra observadas y las reportadas por otros autores, podrían deberse a diferencias entre variedades, o al estado fisiológico de la planta al momento del muestreo, similar a lo encontrado (Happi-Emaga et al., 2008) en las cáscaras del fruto de variedades de musáceas.

En hojas de maralfalfa se han reportado porcentajes de FND y FAD de $62,06 \%$ y $27,04 \%$, respectivamente (Cárdenas et al., 2012), mientras que en pasto Napier (Pennisetum purpureum), cosechado a una edad de noventa días, se determinaron valores de 23,17\% de HEMI y 37,23\% de CEL (Ansah et al., 2010); estos valores aunque difieren en cierto grado de lo determinado para las muestras analizadas (Cuadro 1), podrían indicar que tanto el pseudo-tallo, como las hojas de musáceas pueden constituir, desde el punto de vista bromatológico, un forraje similar a los utilizados actualmente para alimentación de rumiantes.

\section{Extracto etéreo (EE)}

Las cantidades de extracto etéreo (EE) determinadas (Cuadro 1) fueron ligeramente mayores al 1,3\% que indican Subramanian et al. (1988) en las vainas de Musa sapientum, pero inferiores al 4,1\% reportado en pseudo-tallos de Musa cavendishi (Poyyamozhi y Kadrivel, 1986). Asimismo, el valor de 4,27\% de EE determinado en las láminas de la hoja (Cuadro 1) fue mayor al 2,3\% encontrado al analizar el mismo tipo de material de la especie Musa acuminata (Katongole et al., 2008).

Si se compara la cantidad de EE en las muestras analizadas (Cuadro 1), con la de otros forrajes tropicales como el kikuyo (Pennisetum clandestinum), que puede tener 2,14\% (Sánchez et al., 2005), el material no difirió de forma importante y fue inclusive mayor al 1,07\% que según Herrera-Campos et al. (2009) puede tener el pasto Estrella Africana (Cynodon nlemfluensis).

\section{Carbohidratos no fibrosos (CNF)}

Los valores de carbohidratos no fibrosos obtenidos fueron superiores al 23\% (Cuadro 1), lo cual fue mayor al 10,89\% que se determinó (Sampaio et al., 2010) en pasto señal (Brachiaria decumbens Stapf.), y similar al 23,1\% encontrado en heno de un pasto de zona templada indefinido (Schwab et al., 2006). Por lo anterior, y el bajo nivel de proteína determinado, podría considerarse el material estudiado como una fuente energética con potencial para utilizarse en dietas de rumiantes (NRC, 2001).

\section{Correlaciones de los componentes bromatológicos}

Se encontró una relación sobre el $90 \%$ que actúa de forma directamente proporcional entre el contenido de MS y el porcentaje de PC, HEMI y PCIFND (Cuadro 2), estos resultados difirieron de lo observado en otros forrajes tropicales como el pasto humidícola (Brachiaria humidícola (Rendle) Schweick), donde la relación entre la PC y la MS fue estadísticamente significativa, pero inversamente proporcional (ReyesPurata et al., 2009).

La alta relación entre la PC, HEMI y PCIFND (Cuadro 2), sugirió que la mayoría de compuestos 
Cuadro 2. Correlaciones entre las variables bromatológicas de la planta de musáceas (Musa sp.) en un muestreo de quince plantas con su correspondiente nivel de significancia. Cartago, Costa Rica. 2013.

Table 2. Correlations among musacea (Musa sp.) plant bromatological variables in a sampling of fifteen plants with its corresponding level of significance. Cartago, Costa Rica. 2013.

\begin{tabular}{|c|c|c|c|c|c|c|c|c|c|c|c|}
\hline Componente & MS & PC & CEN & FDN & FAD & LIG & CEL & HEMI & $\mathbf{E E}$ & PCIFND & $\mathrm{CNF}$ \\
\hline \multirow[t]{2}{*}{ Materia seca (MS), \% } & 1,00 & 0,92 & $-0,82$ & 0,80 & 0,09 & 0,88 & $-0,52$ & 0,90 & 0,82 & 0,93 & $-0,46$ \\
\hline & & $<0,01$ & $<0,01$ & $<0,01$ & 0,63 & $<0,01$ & $<0,05$ & $<0,01$ & $<0,01$ & $<0,01$ & 0,01 \\
\hline \multirow[t]{2}{*}{ Proteína cruda (PC), \% } & & 1,00 & $-0,62$ & 0,68 & $-0,15$ & 0,82 & $-0,72$ & 0,95 & 0,88 & 0,99 & $-0,47$ \\
\hline & & & $<0,01$ & $<0,01$ & 0,41 & $<0,01$ & $<0,01$ & $<0,01$ & $<0,01$ & $<0,01$ & $<0,01$ \\
\hline \multirow[t]{2}{*}{ Cenizas totales (CEN),\% } & & & 1,00 & $-0,55$ & $-0,05$ & $-0,56$ & 0,34 & $-0,63$ & $-0,57$ & $-0,62$ & 0,04 \\
\hline & & & & $<0,01$ & 0,79 & $<0,01$ & 0,07 & $<0,01$ & $<0,01$ & $<0,01$ & 0,84 \\
\hline \multirow[t]{2}{*}{ Fibra neutro detergente (FDN),\% } & & & & 1,00 & 0,57 & 0,89 & $-0,05$ & 0,75 & 0,74 & 0,72 & $-0,85$ \\
\hline & & & & & $<0,01$ & $<0,01$ & 0,79 & $<0,01$ & $<0,01$ & $<0,01$ & $<0,01$ \\
\hline \multirow[t]{2}{*}{ Fibra ácido detergente (FAD), \% } & & & & & 1,00 & 0,33 & 0,76 & $-0,11$ & $-0,01$ & $-0,12$ & $-0,61$ \\
\hline & & & & & & 0,07 & $<0,01$ & 0,56 & 0,95 & 0,52 & $<0,01$ \\
\hline \multirow[t]{2}{*}{ Lignina (LIG), \% } & & & & & & 1,00 & $-0,36$ & 0,81 & 0,75 & 0,84 & $-0,72$ \\
\hline & & & & & & & 0,05 & $<0,01$ & $<0,01$ & $<0,01$ & $<0,01$ \\
\hline \multirow[t]{2}{*}{ Celulosa (CEL), \% } & & & & & & & 1,00 & $-0,67$ & $-0,53$ & $-0,70$ & $-0,10$ \\
\hline & & & & & & & & $<0,01$ & $<0,01$ & $<0,01$ & 0,58 \\
\hline \multirow[t]{2}{*}{ Hemicelulosa (HEMI), \% } & & & & & & & & 1,00 & 0,91 & 0,96 & $-0,53$ \\
\hline & & & & & & & & & $<0,01$ & $<0,01$ & $<0,01$ \\
\hline \multirow[t]{2}{*}{ Extracto etéreo(EE), \% } & & & & & & & & & 1,00 & 0,89 & $-0,59$ \\
\hline & & & & & & & & & & $<0,01$ & $<0,01$ \\
\hline \multirow{3}{*}{$\begin{array}{l}\text { Proteína cruda incrustada en la } \\
\text { FND, (PCIFND) } \%\end{array}$} & & & & & & & & & & & \\
\hline & & & & & & & & & & 1,00 & $-0,50$ \\
\hline & & & & & & & & & & & $<0,01$ \\
\hline Carbohidratos no fibrosos (CNF), $\%$ & & & & & & & & & & & 1,00 \\
\hline
\end{tabular}

nitrogenados se encontraban ligados a la hemicelulosa en la pared celular en forma, principalmente, de extensina (NRC, 2001), haciendo que la PC en el material no se encontrara disponible de forma inmediata, sino más bien de acuerdo con la velocidad de degradación de la fibra (Valenciaga et al., 2002; Moreira et al., 2003), en este caso, probablemente a la tasa en que la HEMI sea digerida por la flora ruminal. La baja relación entre la PC con la FAD y la CEL, indicó una baja proporción de PC ligada a la FAD, lo cual favorecería el aprovechamiento del nitrógeno de la planta de musáceas, ya que la proteína presente estuvo más relacionada a la fracción $\mathrm{B}$ que a la $\mathrm{C}$, esta última indigestible para el rumiante (NRC, 2001).
En el presente estudio se obtuvo un coeficiente de correlación alto entre la LIG y la HEMI (Cuadro 2), similar a lo mostrado por otros autores en otros forrajes (Varel et al., 1991). Pese a la poca digestibilidad de la LIG (Vargas, 2005), parece no existió repercusión sobre el aprovechamiento de la HEMI por parte del animal, ya que en otros estudios se ha presentado potencialmente un mayor porcentaje de degradación en materiales como la morera (Boschini, 2001), y Bromegrass (Bromus inermis) (Varel et al., 1991). Además, el aumento de la FND, lignina y HEMI de forma proporcional al aumento en la MS (Cuadro 2), puede estar ligado al acúmulo de carbohidratos estructurales, lo cual tiene estrecha relación con el 
hábito de crecimiento de las musáceas, para el cual se requiere de estructuras de soporte para mantener un porte erecto (Avila et al., 2010).

\section{Degradabilidad ruminal}

No se encontraron diferencias significativas entre parcelas $(p>0,05)$ para ninguno de los componentes evaluados en las pruebas de degradabilidad ruminal del banco de guineo negro; asimismo, no se encontraron diferencias en el porcentaje de componentes solubles para ninguna de las secciones del pseudo-tallo $(p>0,05)$, que a su vez fueron mayores a la porción soluble en el peciolo y la lámina de la hoja ( $\mathrm{p}<0,05)$; adicionalmente, estas dos últimas mostraron una fracción degradable mayor que el vástago $(\mathrm{p}<0,05$; Cuadro 3$)$.

Los resultados obtenidos (Cuadro 3) fueron cercanos a los que se indicaron al analizar cáscara de banano, con valores de componentes solubles de 43,62\% (Monçao et al., 2014); pero fueron mayores al 30,9\% que indicaron Keir et al. (1997) en banano, aunque no especificaron claramente el tipo de material utilizado, lo cual podría explicar las diferencias.

$\mathrm{Si}$ se comparan los resultados obtenidos en la presente investigación con los presentados para morera, de alrededor de $30 \%$ en materiales de 56 días de rebrote (Boschini, 2001), se observa una mayor cantidad de la fracción A (soluble) en el vástago y el peciolo, no así en las hojas; situación similar se presenta cuando se compara con lo reportado para diferentes cultivares de sorgo forrajero los cuales en términos generales tuvieron valores de 20-25\% (Vargas, 2005).

La fracción degradable fue estadísticamente mayor en la hoja que en el pseudo-tallo $(\mathrm{p}<0,05)$, mientras que el porcentaje degradable en el peciolo fue intermedio, lo que indica que el vástago se caracteriza por estar formado en mayor proporción por compuestos solubles (Cuadro 3), lo cual probablemente esté relacionado con la composición nutricional y el contenido de carbohidratos no fibrosos determinado en las muestras analizadas (Cuadro 1). Según Monçao et al. (2014), la fracción B (degradable) fue del 23,39\% en cáscara de banano, lo cual fue menor a lo determinado (Cuadro 3); pero Keir et al. (1997), en muestras de la planta de banano, indicaron un $29,7 \%$ de material degradable, que es considerablemente más cercano a lo que se determinó en la presente investigación.

Para el pasto Napier se determinó una degradabilidad ruminal de la fracción B de 46,2\% (Kariuki et al., 2001), el cual fue superior a los valores obtenidos en este estudio en el vástago y el peciolo, pero similar a lo determinado para la lámina (Cuadro 3), demostrando que el pseudo-tallo constituye un material con un comportamiento diferente al de otros forrajes disponibles en condiciones tropicales, cuyo

Cuadro 3. Constantes de degradabilidad ruminal de un muestreo de quince plantas de musáceas (Musa sp.) de acuerdo a la parte de la planta. Cartago, Costa Rica. 2013.

Table 3. Ruminal degradability constants in a sampling of fifteen plants. of musacea (Musa sp.) plant according to part of the plant. Cartago, Costa Rica. 2013.

\begin{tabular}{lcccccccc}
\hline & Fracción & Fracción & & $\begin{array}{c}\text { Velocidad de } \\
\text { degradación } \\
\text { de la fracción } \\
\text { Parte de la planta }\end{array}$ & $\begin{array}{c}\text { Fracción } \\
\text { soluble, \% }\end{array}$ & $\begin{array}{c}\text { degradable, \%/h } \\
\text { potencialmente } \\
\text { degradable en } \\
\text { rumen, \% }\end{array}$ \\
\hline Pseudo-tallo basal & 47,43 & $\mathrm{a}$ & 35,43 & $\mathrm{~b}$ & 0,075 & $\mathrm{a}$ & 82,86 & $\mathrm{a}$ \\
Pseudo-tallo medio & 42,51 & $\mathrm{a}$ & 34,13 & $\mathrm{~b}$ & 0,06 & $\mathrm{ab}$ & 76,64 & $\mathrm{a}$ \\
Pseudo-tallo apical & 44,77 & $\mathrm{a}$ & 33,45 & $\mathrm{~b}$ & 0,06 & $\mathrm{abc}$ & 78,22 & $\mathrm{a}$ \\
Peciolo & 33,34 & $\mathrm{~b}$ & 38,85 & $\mathrm{ab}$ & 0,07 & $\mathrm{ab}$ & 72,19 & $\mathrm{~b}$ \\
Lámina & 18,38 & $\mathrm{c}$ & 45,76 & $\mathrm{ab}$ & 0,02 & $\mathrm{~d}$ & 64,14 & $\mathrm{c}$ \\
\hline
\end{tabular}

Letras diferentes en las filas indican diferencias significativas $(\mathrm{p}<0,05)$ entre las secciones de la planta para una misma constante de degradabilidad / Different leters on rows indicate statistical differences $(<0.05)$ between plant parts for each degradability constant. 
principal aprovechamiento se da por medio de la fermentación bacteriana en el rumen.

En el vástago y el peciolo de la hoja la velocidad de degradación fue desde un $5,8 \%$ /hora hasta un máximo de 7,5\%/hora, mientras que en la lámina fue significativamente menor $(\mathrm{p}<0,05)$ al alcanzar únicamente un $1,6 \%$ /hora, lo que indicó que el material poseía una fracción potencialmente degradable reducida en el rumen, si se compara con el resto de muestras estudiadas (Cuadro 3). Estos valores fueron más altos que lo determinado por Katongole et al. (2008), quienes indicaron una velocidad de degradación ruminal de 4,7\%/h en vainas del pseudotallo; pero más bajos que lo reportado por los mismos autores para la hoja $(3,7 \% / \mathrm{h})$.

La baja degradación de la lámina se pudo deber a la composición de la misma, la cual en primer lugar presenta una capa cuticular importante que normalmente está formada por ceras, carbohidratos, alcoholes, aldehídos y cetonas unidos en una matriz altamente hidrofóbica (Hopkins y Hüner, 2009; MarieMagdeleine et al., 2010), la cual aumentaría el tiempo de rezago a nivel del rumen; y en segundo lugar tiene una alta cantidad de FDN y LIG (Cuadro 1) que reducen la capacidad de penetración de los microorganismos ruminales (Katongole et al., 2008; Marie-Magdeleine et al., 2010), llevando a una disminución en la degradación del material.

En la cáscara de banano se encontró una degradabilidad potencial de 67,01\% (Monçao et al., 2014), mientras que para las hojas de la planta de banano se indicó un valor de alrededor del 50\% (Keir et al., 1997), proporciones que fueron inferiores a lo determinado en el presente ensayo (Cuadro 3), indicando la posibilidad de un mayor aprovechamiento por los rumiantes; inclusive los valores presentados no difirieron en grado importante de lo presentado por Chacón-Hernández y Vargas-Rodríguez (2009) en pasto King Grass (Pennisetum purpureum) quienes reportaron un rango de $89,01 \%$ a $72,57 \%$, lo cual indicó la posibilidad de sustituir forrajes de corte, ya utilizados de forma común, por este tipo de fuentes alimenticias alternativas disponibles.

$\mathrm{Al}$ analizar la digestibilidad in vitro de la materia seca (DIVMS) de dos variedades de musáceas, la diferencia en la degradación luego de un día de incubación encontrada fue poca (Poyyamozhi y Kadrivel, 1986); según las muestras analizadas en este estudio, la degradación de las diferentes partes de la planta de musáceas se dio principalmente durante las primeras veinticuatro horas dentro del rumen, siendo máxima la diferencia en la lámina al pasar de uno a dos días de incubación (Figura 1). Además, según lo mencionado anteriormente sobre la composición de esta parte de la planta, los valores de degradabilidad obtenidos fueron los más bajos, volviéndola un suplemento poco aprovechable, y por lo tanto, menos recomendable para su uso en alimentación animal, al compararla con las restantes partes de la planta y al no cumplir la tasa de degradación mínima de $0,04 \%$ /hora recomendada (Preston, 1986; Katongole et al., 2008).

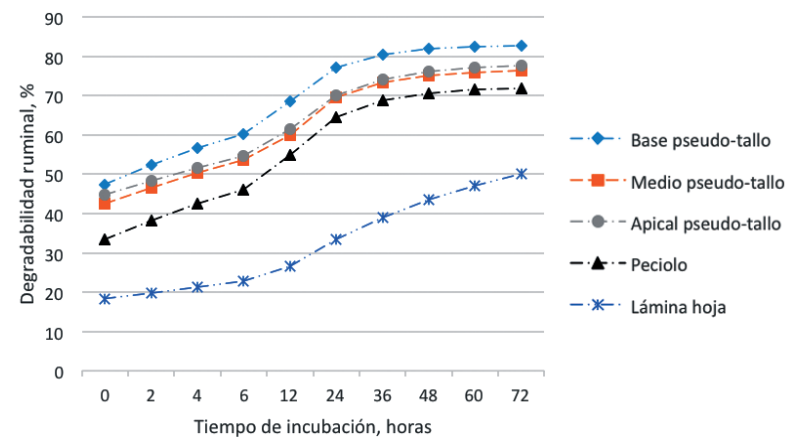

Figura 1. Degradabilidad ruminal según la parte de la planta de las musáceas según una muestra de quince plantas. Cartago, Costa Rica. 2013.

Figure 1. Ruminal degradability according to the part of the musacea plant according to a sampling of fifteen plants. Cartago, Costa Rica. 2013.

La DIVMS de la vaina del plátano (Musa sapientum) fue de $63,4 \%$ en una investigación efectuada por Subramanian et al. (1988), lo cual fue inferior a lo determinado para las tres secciones analizadas del vástago en esta investigación, situación que pudo deberse al método de determinación, ya que Viswanathan et al. (1989) indicaron que el valor de la DIVMS es mayor que el observado por el método in vivo. Comparativamente, Vargas (2005) habló de degradabilidades en un rango de 46\%$64 \%$ al permanecer el material por un lapso de veinticinco horas dentro del rumen (tasa de paso de $4 \%$ /hora), siendo estos valores ligeramente menores que lo determinado para las musáceas (Figura 1). Además, Boschini (2001) en morera, obtuvo un valor de degradabilidad de $69,64 \%$ para la planta entera con una tasa de paso de 4\%/hora, la cual ronda los 
valores señalados para el pseudo-tallo (Figura 1); lo anterior indica que las musáceas pueden sustituir forrajes utilizados comúnmente con porcentajes de aprovechamiento similares.

En términos generales, se considera que la planta de guineo negro (Musa AAA) puede ser utilizadas como alimento para animales rumiantes en los lugares donde el cultivo de la especie vegetal se da de forma regular. A pesar de lo anterior, sumado a su uso deben tomarse en cuenta las características de la misma en términos del bajo contenido de MS en el vástago o palote y la reducida digestibilidad de la lámina de la hoja.

Adicionalmente, la gran cantidad de humedad que contiene el vástago debe ser considerada cuando su uso se realiza en zonas con problemas de sequía durante el verano, pudiendo transformarse el material en una fuente a considerar de suministro de agua al animal.

\section{LITERATURA CITADA}

A.O.A.C. (Association of Official Analytical Chemistry). 2000. Official Methods of Analysis of the Association Analytical Chemistry. 17th Ed. AOAC International. WA, USA.

Amaral, R.C., T.F. Bernardes, G.R. Siqueira, e R.A. Reis. 2008. Estabilidade aeróbia de silagens do CapimMarandu submetidas a diferentes intensidades de compactação ne ensilagem. Rev. Bras. Zootec. 37: 977-983

Ansah, T., E.L.K. Osafo, and H.H. Hansen. 2010. Herbage yield and chemical composition of four varieties of Napier (Pennisetum purpureum) grass harvested at three different days after planting. Agric. Biol. J. N. Am. 1:923-929.

Araya, M., y C. Boschini. 2005. Producción de forraje y calidad nutricional de variedades de Pennisetum purpureum en la meseta central de Costa Rica. Agron. Mesoam. 16:37-43.

Avila, R.E., O.N. Di Marco, M.G. Agnusdei, y C. Mayoral. 2010. Digestibilidad de la fibra y materia seca de dos gramíneas megatérmicas (Chloris gayana y Cenchrus ciliaris) de diferente porte: relación con la edad y largo foliar. Rev. Argentina Prod. Anim. 30:1-13.

Aziz, N.A.A., L.-H. Ho, B. Azahari, R. Bhat, L.-H. Cheng, y M.N.M. Ibrahim. 2011. Chemical y functional properties of the native banana (Musa acuminata $\mathrm{x}$ Balbisiana colla cv. Awak) pseudo-stem tender core flours. Food Chem. 128:748-753.
Boschini, C. 2001. Degradabilidad in situ de la materia seca, proteína y fibra del forraje de morera (Morus alba). Agron. Mesoam. 12:79-87.

Boschini, C., y J. Elizondo. 2005. Determinación de la calidad y la producción de sorgo negro forrajero (Sorghum almun) en edades para ensilar. Agron. Mesoam. 16:29-36.

Boschini-Figueroa, C. 2006. Nutrientes digeribles, energía neta y fracciones proteicas de la morera (Morus alba) aprovechables. Agron. Mesoam. 17:141-150.

Cárdenas, L.R., R. Pinto-Ruíz, F.J. Medina, F. Guevara, H. Gómez, A. Hernández, y J. Carmona. 2012. Producción y calidad del pasto Maralfalfa (Pennisetum sp.) durante la época seca. Quehacer Científico en Chiapas 1:38-46.

Chacón-Hernández, P.A., y C.F. Vargas-Rodríguez. 2009. Digestibilidad y calidad del Pennisetum purpureum cv. King Grass a tres edades de rebrote. Agron. Mesoam. 20:399-408.

Chacón-Hernández, P.A., y C.F. Vargas-Rodríguez. 2010. Consumo de Pennisetum purpureum cv. King Grass a tres edades de cosecha en caprinos. Agron. Mesoam. 21:267-274

Chacón-Hernández,P. 2008. Estimación del aprovechamiento en ganado caprino del Pennisetum purpureum cv. King grass cosechado a tres diferentes edades de rebrote. Agron. Mesoam. 20:399-408.

Costa, K.A.P., R.L. Assis, K.C. Guimarães, E.C. Severiano, J.M.A. Neto, W.S. Crunivel, J.F. Garcia, and N.F. Santos. 2011. Silage quality of Brachiaria brizantha cultivars ensiled with different levels of millet meal. Arq. Bras. Med. Vet. Zootec. 63:188-195.

De-la-Cruz, J.C., y G.A. Gutiérrez. 2006. Alimentación de bovinos con ensilado de mezclas de banano de rechazo y ráquis en diferentes proporciones. Av. Investig. Agropecu. 10(3):29-39.

Dormond, H., A. Rojas, C. Jiménez, y G. Quirós. 2000. Efecto de niveles crecientes de seudotallo de guineo en combinación con ensilaje de maíz, sobre el crecimiento de terneras Jersey, durante la época seca. Agron. Costarricense 24(2):31-40.

García, A., y R.M. Martínez. 1999. Uso de follajes del plátano en la alimentación del cerdo. Rev. Comp. Prod. Porcina 6(3):15-29.

Goering, H.K., and P.J. Van-Soest. 1970. Forage fiber analysis (Apparatus, reagents, procedures and some applications). ARS-USDA, WA, USA.

Happi-Emaga, T., C. Robert, S.N. Ronkart, B. Wathelet, and M. Paquot. 2008. Dietary fibre components and pectin 
chemical features of peels during ripening in banana and plantain varieties. Bioresour. Technol. 99:4346-4354. doi:http://dx.doi.org/10.1016/j.biortech.2007.08.030.

Hernández, J.B., A.E. Cañizares-Chacín, G. Blanco, I. Arrieche, A. Pérez, C. Salazar, y M. González. 2009. Contenido de nitrógeno, fósforo y potasio en harinas de clones de musáceas comestibles (Musa spp.). Rev. Cient. UDO Agríc. 9:449-457.

Herrera-Campos, L., C.F. Vergas-Rodríguez, C. BoschiniFigueroa, y A. Chacón-Villalobos. 2009. Variación bromatológica de la leche de cabras Lamancha alimentadas con diferentes forrajes. Agron. Mesoam. 20:381-390.

Hopkins, W.G., and N.P.A. Hüner. 2009. Introduction to plant physiology. $4^{\text {th }}$ ed. John Willey \& Sons, Inc, N.Y. USA.

Kariuki, J.N., S. Tamminga, C.K. Gachuiri, G.K. Gitau, and J.M.K. Muia. 2001. Intake and rumen degradation in cattle fed napier grass (Pennisetum purpureum) supplemented with various levels of Desmodium intortum and Ipomoea batatas vines. S. Afr. J. Anim. Sci. 31:149-157.

Katongole, C.B., F.B. Bareeba, E.N. Sabiiti, y I. Ledin. 2008. Nutritional characterization of some tropical urban market crop wastes. Anim. Feed Sci. Technol. 142:275-291.

Keir, B., N. Van Lai, T.R. Preston, and E.R. Orskov. 1997. Nutritive value of leaves from tropical trees and shrubs: 1 . In vitro gas production and in sacco rumen degradability. Livest. Res. Rural Dev. 9:1-8.

Marie-Magdeleine, C., M. Boval, L. Philibert, A. Borde, and H. Archimède. 2010. Effect of banana foliage (Musa $x$ paradisiaca) on nutrition, parasite infection and growth of lambs. Liv. Sci. 131:234-239.

Maurice, I., F. Gastal, and J.-L. Durand. 1997. Generation of form and associated mass deposition during leaf development in grasses: A kinematic approach for non-steady growth. Ann. Bot. 80:673-683.

Mazzeo, M., L. León, L.F. Mejía, L.E. Guerrero, y J.D. Botero. 2010. Aprovechamiento industrial de residuos de cosecha y poscosecha del plátano en el departamento de Caldas. Rev. Educación en Ingeniería 9:128-139.

Mohapatra, D., S. Mishra, and N. Sutar. 2010. Banana and its by-product utilisation: An overview. J. Sci. Ind. Res. 69:323-329.

Monçao, F.P., S.T. Reis, J.P.S. Rigueira, E.C.J. Sales, A.P.S. Antunes, E.R. Oliveira, e Z.G. Carvalho. 2014. Degradabilidade ruminal da matéria seca e da FND da casca de banana tratada com cal virgem. Rev. Ciências Agrárias 37:42-49.

Moreira, F.B., I.N. do-Prado, U. Cecato, F. Yoshimi, W.G. do-Nascimento, N.E. e Souza. 2003. Suplementação com sal mineral proteinado para bovinos de corte, em crescimento e terminação, mantidos em pastagem de grama estrela roxa (Cynodon plectostachyus Pilger), no Inverno. Rev. Bras. Zootec. 32:449-455.

NRC (National Research Council). 2001. Nutrient requirements of dairy cattle. 7 Rev, National Academy Press, WA, USA.

Oliveira, L., N. Cordeiro, D.V. Evtuguin, I.C. Torres, and A.J.D. Silvestre. 2007. Chemical composition of different morphological parts from "Dwarf Cavendish" banana plant and their potential as a nonwood renewable source of natural products. Industrial Crops and Products 26:163-172.

Ørskov, E.R., F.D.D. Hovell, and F. Mould. 1980. The use of the nylon bag technique for the evaluation of feedstuffs. Trop. Anim. Prod. 5:195-213.

Osuji, J., G. Harrison, J. Heslop-Harrison, J. Osuji, J. Crouch, and J. Crouch. 1997. Identification of the genomic constitution of Musa L. lines (bananas, plantains and hybrids) using molecular cytogenetics. Ann. Bot. 80:787-793.

Poyyamozhi, V.S., and R. Kadrivel. 1986. The value of banana stalk as a feed for goats. Anim. Feed Sci. Technol. 15:95-100.

Preston, T.R. 1986. Better utilization of crop residues and by-products in animal feeding: Research guidelines. 2. A practical manual for research workers. FAO, Roma, ITA.

Rabemanolontsoa, H., and S. Saka. 2012. Holocellulose Determination in Biomass. Zero-Carbon Energy Kioto 2011. Springer, Kioto, JPN.

Reyes-Purata, A., E.D. Bolaños-Aguilar, D. HernándezSánchez, E.M. Aranda-Ibañez, y F. Izquierdo-Reyes. 2009. Producción de materia seca y concentración de proteína en 21 genotipos del pasto Humidícola (Brachiaria humidícola (Randle) Schweick). Universidad y Ciencia 25:213-224.

Sampaio, C.B., E. Detmann, M.F. Paulino, S.C. Valadares Filho, M. A. de Souza, L.I. azzarini, P.V.R. Paulino, and A.C. de Queiroz. 2010. Intake and digestibility in cattle fed lowquality tropical forage and supplemented with nitrogenous compounds. Trop. Anim. Health Prod. 42:1471-9.

Sánchez, D.E., L. Arreaza, y B. Abadía. 2005. Estudio de la cinética de degradación in vitro de cuatro forrajes 
tropicales y una leguminosa de clima templado. Rev. CORPOICA 6(1):58-68.

Sánchez, J.M., L. Piedra, y H. Soto. 1998. Calidad nutricional de los forrajes en zonas con niveles bajos de produccion de leche, en la zona norte de Costa Rica. Agron. Costarricense 22(1):69-76.

SAS. 1985. SAS User's guide: Statistics. Versión 5 ed. SAS Institute Inc. Cary. N.C., USA.

Schwab, E.C., C.G. Schwab, R.D. Shaver, C.L. Girard, D.E. Putnam, and N.L. Whitehouse. 2006. Dietary forage and nonfiber carbohydrate contents influence B-vitamin intake, duodenal flow, and apparent ruminal synthesis in lactating dairy cows. J. Dairy Sci. 89: 174-87.

Steel, R., y J. Torrie. 1988. Bioestadística: principios y procedimientos. McGraw Hill, México DF, MEX.

Subramanian, P.R., R. Kadrivel, K. Viswanathan, and D. Chandrasekaran. 1988. In vitro studies and short-term feeding trial in lambs to evaluate plantain sheath (Musa sapientum) as a feed for ruminants. Anim. Feed Sci. Technol. 20:343-348.

Valenciaga, D., B. Chongo, e I. Scull. 2002. Caracterización del clon Pennisetum CUBA CT-115. Fraccionamiento proteico y degradabilidad ruminal del nitrógeno. Rev. Cub. Cienc. Agric. 36:259-264.

Van-Soest, P.J., J.B. Robetson, and B.A. Lewis. 1991. Methods for dietary fiber, neutral detergent fiber and non starch polysaccharides in relation to animal nutrition. J. Dairy Sci. 74:3583-3597.
Varel, V.H., H.G. Jung, and L.R. Krumholz. 1991. Degradation of cellulose and forage fiber fractions by ruminal cellulolytic bacteria alone and in coculture with phenolic monomer-degrading bacteria. J. Anim. Sci. 69:4993-5000.

Vargas, C.F. 2004. Valoración de parámetros nutricionales y degradabilidad ruminal de 15 ecotipos de sorgo forrajero (Sorghum sp). Universidad de Costa Rica, San José, CRC.

Vargas, C.F. 2005. Degradabilidad ruminal de genotipos de sorgo. Agron. Mesoam. 16:215-223.

Vásquez, A. 1982. Estudio detallado de los suelos de la Estación Experimental de Ganado Lechero El Alto. Facultad de Ciencias Agroalimentarias. Escuela de Fitotecnia. Universidad de Costa Rica, San José, CRC.

Villalba, D.K., V.A. Holguín, J.A. Acuña, y R.P. Varón. 2011. Calidad bromatológica y organoléptica de ensilajes de residuos orgánicos del sistema de producción café musáceas. Rev. Colomb. Cienc. Anim. 4(1):47-52.

Viswanathan, K., R. Kadrivel, and D. Chyrasekaran. 1989. Nutritive value of banana stalk (Musa cavendishi) as a feed for sheep. Anim. Feed Sci. Technol. 22:327-332.

Weiss, W.P., H.R. Conrad, and N.R. St. Pierre. 1992. A theoretically-based model for predictong total digestible nutrient values of forages and concentrates. Anim. Feed Sci. Technol. 39:95-110. 